\title{
Experimental Evidence for Electromagnetic Coupling Inhomogeneity Along the Grain Boundary Plane in High Angle Melt-Textured $\mathrm{YBa}_{2} \mathrm{Cu}_{3} \mathrm{O}_{6+\mathrm{x}}$ Bicrystals
}

\author{
Michael B. Field, Alex Pashitski, Anatoli Polyanskii, and David C. Larbalestier \\ Applied Superconductivity Center and Materials Science Program, University Of Wisconsin-Madison, WI 53706 USA \\ Apurva S. Parikh and Kamel Salama \\ Texas Center for Superconductivity and Mechanical Engineering Department, University of Houston, TX 77204 USA
}

\begin{abstract}
Detailed characterization of two high angle general misorientation $\mathrm{YBa}_{2} \mathrm{Cu}_{3} \mathrm{O}_{6+\mathrm{x}}$ bicrystals produced by the melt-texture-liquid-phase-removal method shows that the boundaries contain regions of both strong and weak coupling. In one bicrystal the strongly coupled component was inferred from analysis of the magnetic-field-dependent voltage-current characteristics and confirmed with magneto-optical images of the boundary. Several sections of the second sample having macroscopically different grain boundary facets were tested after sectioning with a laser. The properties were not dependent on the grain boundary plane orientation. Highfield, high-sensitivity voltage-current characteristics of the inter and intra-grain regions had qualitatively identical properties and confirmed that there was a substantial strong coupled component to the boundary. The data confirm that the melt-texture-liquid-phase-removal method of $\mathrm{YBa}_{2} \mathrm{Cu}_{3} \mathrm{O}_{6+1}$ formation is beneficial to the formation of strong-coupling regions in high angle grain boundaries and that some high angle grain boundaries contain strongly coupled components.
\end{abstract}

\section{INTRODUCTION}

General knowledge of the misorientation dependence of the electromagnetic coupling of $\mathrm{YBa}_{2} \mathrm{Cu}_{3} \mathrm{O}_{6+x}$ (YBCO) bicrystals comes mainly from the study of thin film bicrystals [1] where the misorientation relationships are generally constrained to be simple. By contrast the misorientation relationships for polycrystals are much more general. The strength of the electromagnetic coupling as a function of grain boundary misorientation in melt-textured $\mathrm{YBa}_{2} \mathrm{Cu}_{3} \mathrm{O}_{6+\mathrm{x}}$ is accordingly less well understood. Many bulk scale grain boundaries lack a unique boundary plane, since the plane may wander and in they also exhibit both macroscale and microscale facets. Thus the typical bulk sample grain boundary does not follow any particular low index plane and their properties may be expected to be variable and more complex than epitaxial thin film bicrystals.

In the earlier study of high angle melt-textured bicrystals by Field et al. [2], we compared the $J_{c}(B)$ character of several samples whose misorientation varied. All samples showed signs of weakly coupled regions but there was also a marked decrease in the $\mathrm{J}_{c}(B)$ sensitivity with decreasing

Manuscript received October $17,1994$.

This work was supported by the NSF MRG (DMR-9214707) misorientation angle and, for some samples, a residual, weakly field dependent supercurrent was present even at $7 \mathrm{~T}$ and $77 \mathrm{~K}$. It was speculated that the residual current came from a minority strongly coupled component. A recently introduced modification to the processing of melt-textured samples by Parikh et al. [3] has been reported to greatly increase the intergrain $J_{c}$ values of high angle bicrystals. This process melts out a significant fraction of the liquid phase, perhaps removing constituents which degrade the coupling strength of the grain boundary.

The observation of a residual supercurrent in bicrystals which exhibit strong sensitivity to low fields has attracted much attention and it is widely thought that their grain boundaries contain parallel strongly and weakly coupled paths $[4,5,6,7]$. A second idea is that the residual current is a property of inhomogeneous large Josephson junctions [8]. A third possibility is [9] that the residual supercurrent is the result of Dayem bridges in the grain boundary. None of these various models have been explicitly verified, nor is the boundary plane dependence of the properties clear.

The goal of the present work was to investigate the coupling strength of high angle grain boundaries in melt textured samples made by this new melt-out method. We studied our samples by transport and magneto-optical [10] methods.

\section{EXPERIMENTAL PROCEDURE}

Samples were made by the liquid phase removal or "melt out" technique [3] a process in which some $40 \%$ of the liquid phase is melted out into a porous $\mathrm{Y}_{2} \mathrm{BaCuO}_{5}(211$ phase) block. Excess $\mathrm{CuO}$ and $\mathrm{BaCuO}_{2}$ phases that might contaminate the grain boundary are believed to leave the YBCO sample during this step. Two parent bar-shaped samples were cut from the melt, KS1B1 and KS4B1. The bars were carefully ground and polished to less than $100 \mu \mathrm{m}$ thickness so that the grain structure could be observed from both sides. Samples $\sim 300 \mu \mathrm{m}$ wide were produced from the ground bars by slicing with a pulsed Nd-YAG laser ( $\lambda=106.4 \mathrm{~nm}$, frequency $250 \mathrm{~Hz}$, pulse width $15 \mathrm{~ns}$ ) having beam sizes of $2-20 \mu \mathrm{m}$ dia. and an energy density up to $40 \mathrm{~J} / \mathrm{cm}^{2}$. Laser cutting allowed the selection of bicrystals from the polycrystalline starting material, and also helped lower the measuring currents needed to determine $I_{c}$. An 
earlier study of laser cutting of YBCO put the upper limit of the damaged region to less than $3 \mu \mathrm{m}$ for a laser of the same wavelength and two orders of magnitude more power [11]. Damage near the cut edges could not be detected by light microscopy (resolution $\sim 1 \mu \mathrm{m}$ ).

The misorientation of the two grains in each bicrystal was determined quantitatively by $\mathrm{x}$-ray pole figure analysis. Information on the location of the (006), (013/103), and $(020 / 200)$ poles was used to determine the direction cosines of the elements of a misorientation matrix. Given this matrix, one can either express the misorientation in terms of Euler angles or the one-axis, one-angle misorientation scheme [12]. The former permits a more direct visualization of the misorientation, while the latter reduces the mixed misorientation angles to one value (Fig. 1). In the tetragonal system (to which twinned YBCO is approximated) there are 8 equivalent axis/angle pairs to describe any misorientation, all related by symmetry. The smallest of the 8 misorientation angles is referred to as the "disorientation" angle, and by convention this axis/angle pair is chosen to describe the misorientation. In terms of Euler angles, KS1B1 had $\phi=13^{\circ}, \varphi_{1}=0^{\circ}, \varphi_{2}=77^{\circ}$ and KS4B1 had $\phi=5^{\circ}$, $\varphi_{1}=102^{\circ}, \varphi_{2}=101^{\circ}$. In terms of the axis/angle scheme, KS1B1 had $\theta=14^{\circ}$ about $[0.8310 .1050 .184] \sim\left[\begin{array}{lll}24 & 3 & 5\end{array}\right]$, and KS4B1 had $\theta=11^{\circ}$ about [0.234 $\left.0.054-0.235\right] \sim\left[\begin{array}{lll}13 & 3 & -13\end{array}\right]$. Both bicrystals should thus be considered to contain high angle grain boundaries according to the definition of Dimos et al. (misorientations greater than $5-10^{\circ}$ of any type) [1].

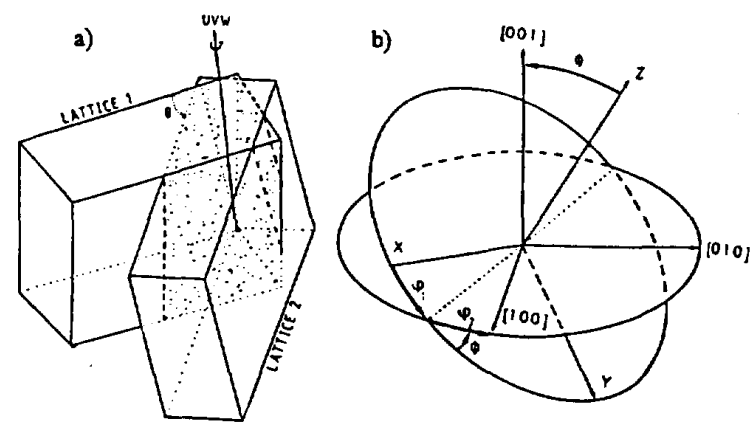

Fig. 1. Two methods to describe crystal misorientation from a misorientation matrix. a) One-pole, one-axis scheme: the misorientation angle $\theta$ is defined about an axis UVW that is common to both lattices. b) The Euler angle description utilizes 3 angles relating the Cartesian coordinates of the 2 grains.

After cutting and polishing, samples were mounted on sapphire substrates and current and voltage leads of 25 to $50 \mu \mathrm{m}$ dia. were attached within the grains and across the boundary [2]. To prevent sample burnout, pulsed currents were used for most of the higher current data. A Philips PM 5139 function generator was used to drive a Kepco bipolar power supply to produce $1 \mathrm{~ms}$ pulses of currents up to $8 \mathrm{~A}$ with a $10 \mathrm{~Hz}$ duty cycle. The pulses had a $200 \mu$ s flat-top measuring window in order to minimize the inductive component to the measured voltage. However, the relatively high noise level $( \pm 2.5 \mu \mathrm{V})$ required a large number of points for the data to be satisfactorily smoothed. DC data of higher sensitivity could be taken with a Keithley 2001 voltmeter and 1801 nanovolt preamplifier at lower currents $(<0.5 \mathrm{~A})$ without risk to the current leads or contacts. The noise level was then $\approx 10 \mathrm{nV}$. There was excellent agreement in the overlap region between the pulsed and DC measurements.

The magneto-optic (MO) images were taken in a polarized light microscope using a Bi-doped yttrium iron garnet film placed directly on the sample (KS1B1a.2). Sample and film were cooled in a continuous flow cryostat and viewed at temperatures down to $\sim 10 \mathrm{~K}$ in fields up to $100 \mathrm{mT}$. Images were recorded by videotape and computer.

\section{RESULTS}

\section{A. Effect of the Macroscopic Grain Boundary Plane}

Sample KS4AB1 had a curved grain boundary which was subdivided into 3 subsamples $K S 4 A B 1 a, b$, and c. KS4AB la and $b$ had similar grain boundary planes, which were inclined at $\sim 45^{\circ}$ to the long length of the sample in the direction of current flow (Fig 2a,b). The boundary plane in KS4AB 1c bends so as to become nearly normal to the direction of current flow (Fig. 2c). Because the grain boundary plane was in the same region at top and bottom, we believe that the macroscopic facet is essentially straight through the sample.

The critical currents of all the sub-samples of KS4AB1 used for the macrofacet experiment exhibited a decline of $-70-85 \%$ in fields of $\sim 20 \mathrm{mT}$ (Fig. 3). All sub-samples exhibited a similar residual supercurrent of $1-2 \times 10^{3} \mathrm{~A} / \mathrm{cm}^{2}$, which was independent of field in the range 20 to $400 \mathrm{mT}$.

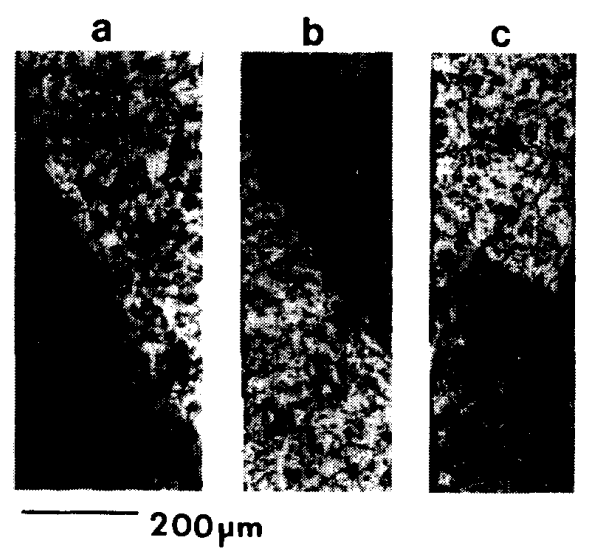

Fig 2. Polarized light micrographs of samples KS4B1 a,b, and c. 


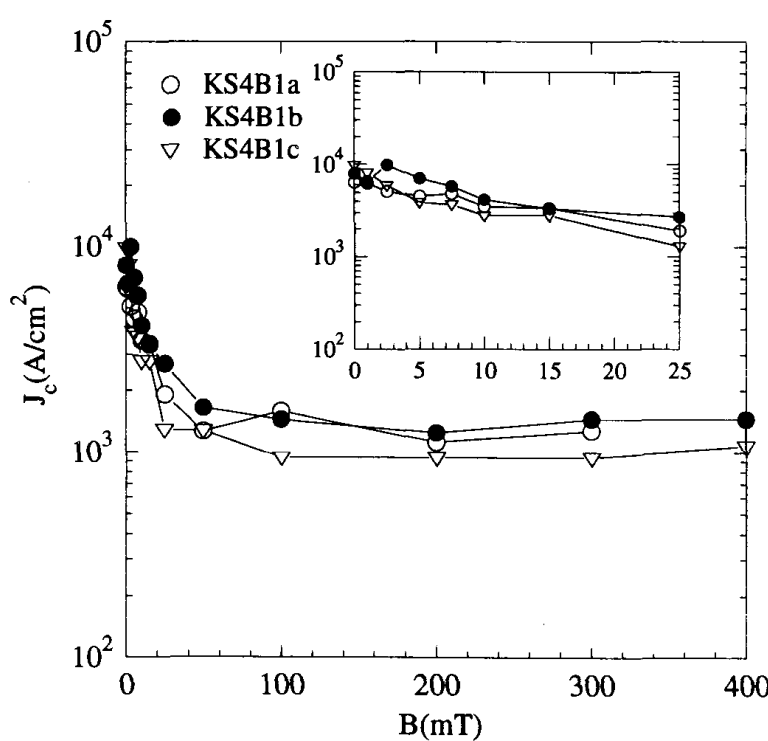

Fig. 3. J $\mathrm{J}_{\mathrm{c}}(\mathrm{B})$ traces for sub samples KS4B1a,b, and $\mathrm{c}$ at $77 \mathrm{~K}, \mathrm{~B} \sim \perp \mathrm{c}$ Most of the decline in $\mathrm{J}_{\mathrm{c}}$ occurs below $50 \mathrm{mT}$. The behavior of the three samples is qualitatively similar.

\section{B. Magneto-optic Imaging}

Magneto-optic imaging was used on sample KS1B1a.2 because of its bimodal V-I characteristics (Fig. 4). These exhibit clear signs of parallel weakly and strongly coupled

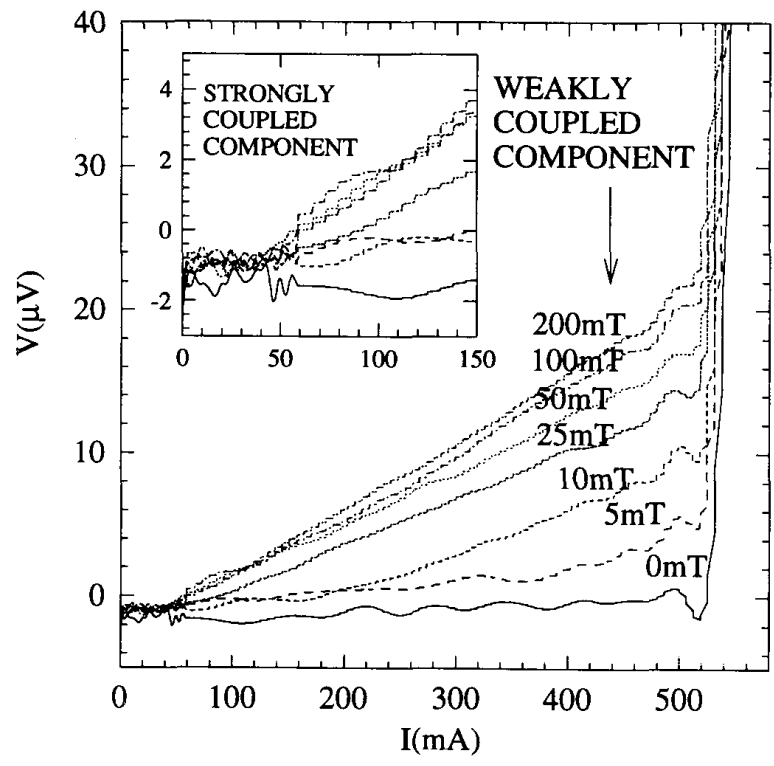

Fig. 4. Pulsed V-I (B) traces of sample KS1B1a.2 at $77 \mathrm{~K}$. A fieldindependent residual current of $\sim 50 \mathrm{~mA}$ is visible.

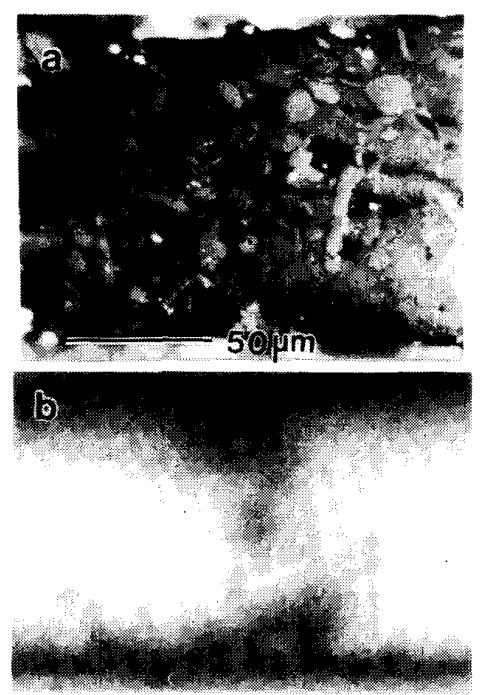

Fig. 5. a) Polarized light micrograph of the grain boundary in sample KS1B1a.2. b) Magneto-optic image of the same region. The image was taken after being field cooled to $8 \mathrm{~K}$ in $40 \mathrm{mT}$ with an additional in plane field to induce current to cross the boundary.

components, the strongly coupled component being the minority component with the field-independent trace. In an attempt to explore the strongly coupled region further, we imaged it. Fig. 5 shows that flux preferentially entered the boundary except for a bridge $\sim 15 \mu \mathrm{m}$ wide which resisted flux entry (after field cooling in $40 \mathrm{mT}$ to $8 \mathrm{~K}$ ) in a manner similar to that of the intragranular regions. Thus, at least in this case, the parallel strongly coupled component had a macroscopic component much larger than any of the characteristic lengths of the superconducting state.

\section{High Field High Sensitivity V-I Traces}

High sensitivity V-I measurements were taken on sample KS4B1b in fields from $1 \mathrm{~T}$ up to $6 \mathrm{~T}$. The sample was mounted so that $\mathrm{H}$ was $\sim$ llc. Fig. 6 shows that the curvature of the V-I traces changed between 4.5 and $4.75 \mathrm{~T}$, thus signaling the irreversibility line in grain "B". Similar plots for the grain boundary are shown in Fig. 7. The transition in this case occurred at $\sim 5 \mathrm{~T}$, indicating that the grain boundary remained in a flux pinning state to a slightly higher field than the grain interior. This is unambiguous evidence that a significant component of this high angle grain boundary had similar flux pinning.properties to the grain interior.

\section{Discussion}

The present data support the claim that the melt out method of melt texturing can produce strongly coupled high angle grain boundaries. Both samples exhibited the characteristics of mixed behavior but both also had 


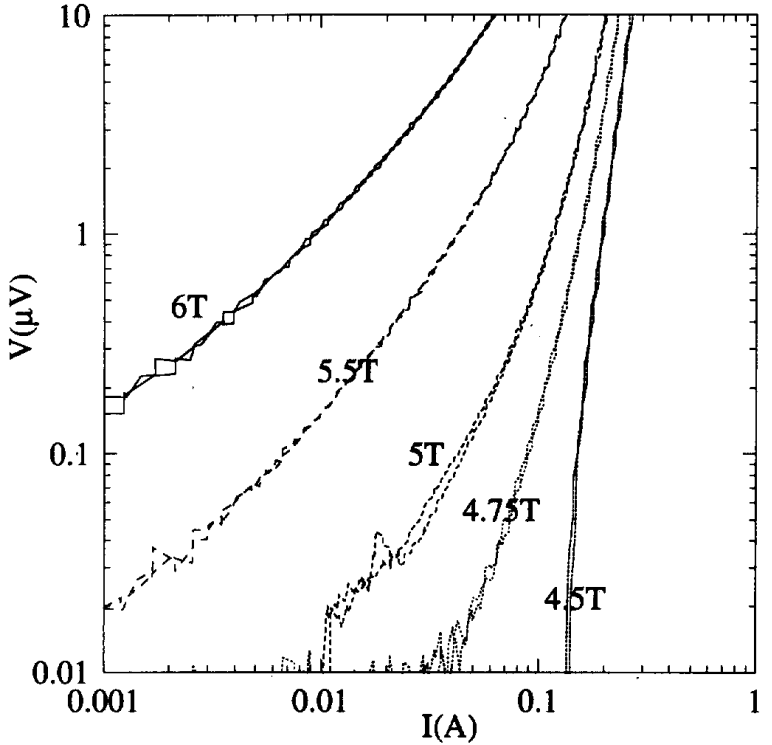

Fig. 6. Intragrain logarithmic V-I characteristics for grain "B" in KS4B 1b as a function of magnetic field at $77 \mathrm{~K}$., B $-\| c$ Note that the irreversibility field is between $4.5-4.75 \mathrm{~T}$.

characteristics that are found in strongly coupled grain boundaries. For sample KS1B1, the evidence was in the field dependence of the $J_{c}$ and in the MO image. The MO image showed that the strong coupled section was macroscopic in scale, being $>10 \mu \mathrm{m}$ in width. However, the strong pinning bridge does not appear to be a related to any

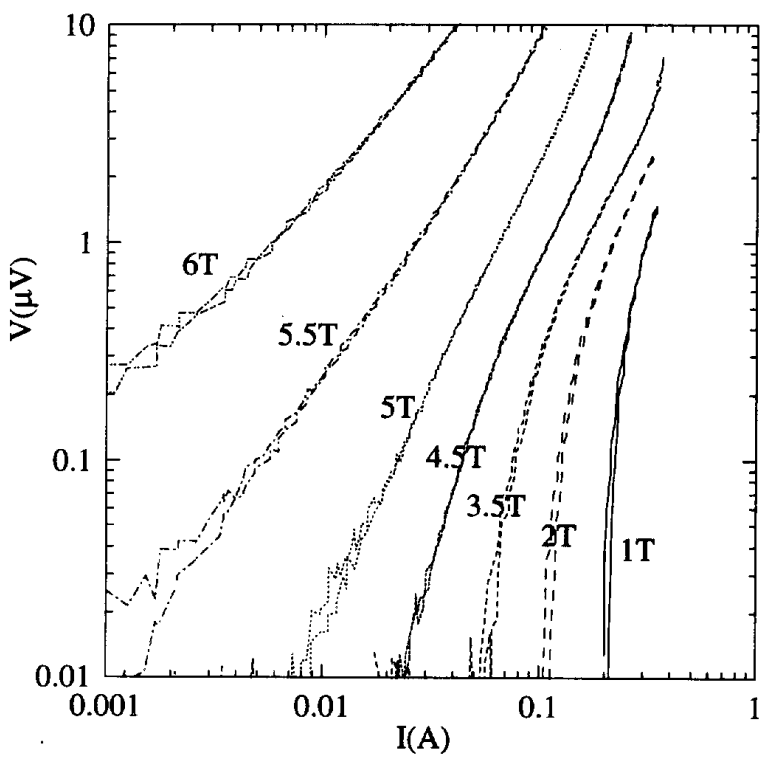

Fig. 7. Log V-I across the grain boundary in KS4B 1b as a function of high magnetic field at $77 \mathrm{~K}, \mathrm{~B} \sim \mathrm{ll}$. Note the irreversibility line is at about $5 \mathrm{~T}$. specific macroscopic feature of the grain boundary. For sample KS4B1b the position of the strong coupled section has not yet been identified but the extended V-I characteristics show that the grain boundary resisted thermal depinning a little better than one of the grains!

An additional significant point is that the subsectioning of sample KS4B1 into 3 samples having a different boundary plane had no effect on the qualitative $J_{c}(B)$ character, and only a minor effect on the quantitative $J_{c}$ values.

\section{CONCLUSION}

We have found strong evidence for electromagnetic coupling inhomogeneity across grain boundaries in high angle, liquid-phase removal melt-processed YBCO via magneto-optic and current transport experiments. In one sample, a magneto-optic technique was used to observe a strongly-coupled path across the grain boundary. Another sample was subsectioned to demonstrate that the orientation of the grain boundary plane did not have a noticeable effect on the coupling across a high angle grain boundary for different sections having the same crystal misorientation. The residual critical current seen at high magnetic fields is proposed to be of a flux-pinning nature, supported by the evidence that the irreversibility line across the grain boundary is about the same as in the grains.

\section{ACKNOWLEDGMENT}

We would like to thank Harry Edelman for developing the pulsed current measuring system and Alex Squitieri for assistance with the high field measurements. Many discussions with X. Y. Cai and S. E. Babcock are gratefully appreciated.

\section{REFERENCES}

[1] D. Dimos, J. Mannhart and P. Chaudhari, Phys. Rev. B, vol 41, pp 4038-4049, March 1990.

[2] M.B. Field, X.Y. Cai, S.E. Babcock, and D.C. Larbalestier, IEEE Trans on Superconductivity, vol.3(1), pp. 1479-1482, 1993.

[3] A.S. Parikh, B. Meyer, and K. Salama, Supercond. Sci. Technol., vol 7 pp. $455-461,1994$

[4] J.W. Ekin, H.R. Hart, Jr. and A.R. Gaddipati, J. Appl. Phys., Vol 68(5), pp. 2285-2295, 1990.

[5] M.B. Field, U of Wisconsin-Madison Preliminary Examination, January 1994.

[6] D. Shi, J of Electronic Materials, vol. 22(10), pp. 1211-1219, 1993

[7] C. Sarma, G. Schindler, D.G. Haase, C.C. Koch, A.M. Saleh, and A.I Kingon, Appl. Phys. Lett., vol 64(1), pp.109-111, January 1994.

[8] I.K. Yanson, Soviet Physics JETTP, vol 31, p. 800, 1970

[9] E. Sarnelli, P. Chaudhari and J. Lacy, Appl. Phys. Lett., vol 62, pp. 777, 1993.

[10] L.A. Dorosinskii, M.V. Indenbom, V.I. Nikitenko, Yu.A. Ossip'yan, A.A. Polyanskii, V.K. Vlasko-Vlasov, Physica C, vol. 203, pp. 149-156, 1992.

[11] U. Varshney, B. D. Eichelberger III, J. M. Glass, R. J. Churchill, A. I. Kingon, J Mater. Res., vol. 8(10), pp. 2429-2432, 1993.

[12] V. Randle, "The Measurement of Grain Boundary Geometry," Institute of Physics Publishing, Bristol UK, 1993. 